\title{
A hidden catalysis: metal-, and organocatalyst-free one-pot assembly of chiral aza-tricyclic molecules
}

\author{
Dung Tien Do ${ }^{*}$,b \\ ${ }^{a}$ Faculty of Chemistry, University of Science, Vietnam National University-Hanoi, 19 Le Thanh Tong, Hoan Kiem, Ha Noi, \\ Vietnam \\ ${ }^{b}$ School of Pharmacy, Connecticut University, Storrs, Connecticut, United State
}

E-mail: dtdung82@hus.edu.vn

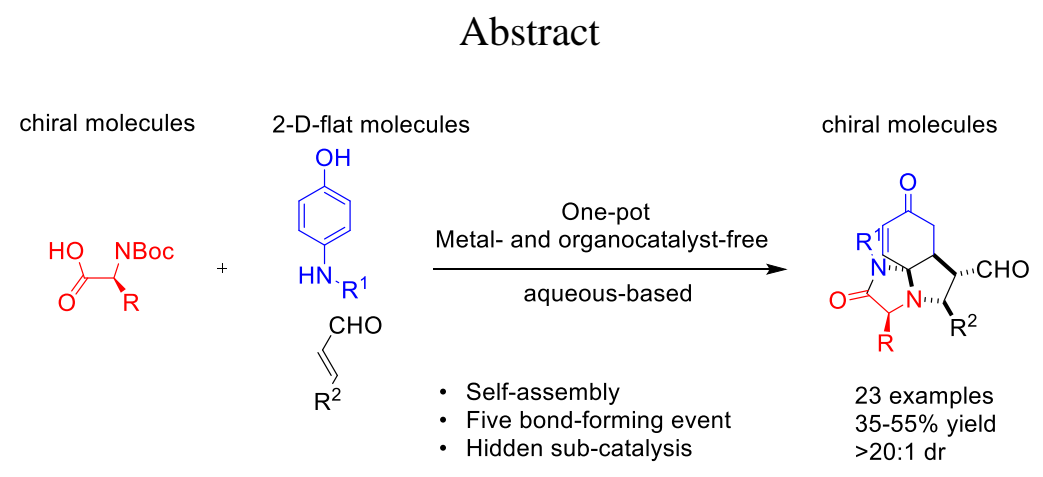

Development of a rapid synthesis of complex molecules from simple building blocks under a metal-and organocatalyst-free condition is both conceptually and chemically challenging. Here, we developed a hidden catalysis that allow the straightforward assembly of enantiopure aza-tricyclic molecules containing six contiguous stereocenters from aminophenols, $\alpha, \beta$-unsaturated aldehydes and $\alpha$-amino acids. Without using a metal or an organocatalyst, our approach relies on a temporary formation of a spiroimidazolidinone intermediate and its participation in a sequential aza-Michael/Michael reaction as both a substrate and a catalyst under an iminium/enamine catalysis. The formation of the putative iminium intermediate was supported by spectroscopic data and its interruptive reduction derivative was isolated and fully characterized. Whereas a conventional catalyst is always present and does not undergo a permanent chemical change in a classic catalysis, the spiroimidazolidinone intermediate is conceptualized as a sub-catalyst as it is only temporary produced from precursors and catalyzes its own consumption. This unique substrate-catalyst (sub-catalyst) dual role of the spiroimidazolidinone induces a substantial steric discrimination in the transition state and an excellent overall diastereoselectivity ( $>20: 1 \mathrm{dr}$ ). It allows the use of an amino acid precursor as the sole chirality genesis and avoids the use of transition metals or organocatalysts. An enantiomer of an aza-tricyclic imidazolidinone can be prepared from a commercially available amino acid precursor. The aqueous-based reaction is practical and scalable for multi-gram synthesis. The success of implementing this sub-catalysis concept in the synthesis will pave the way for many efficient chiral catalyst-free preparations of chiral complex molecules. 


\section{Introduction}

Chiral molecules with their defined 3-D structures are of paramount importance for the study of chemical biology and drug discovery. Nature's ability of rapid assembly of molecules with structural diverse at various levels of complexity has inspired numerous development of efficient synthetic methodologies. ${ }^{1,2}$ In practice, chiral architectures are usually prepared from organometallic and organocatalytic processes where transition metals or enzymes or organocatalysts are operated for a desired reaction. Whereas enzymatic catalysis sets a high standard for the stereoselectivity, organometallic- and organo-catalysis have an advantage for a more diverse scope of reactions. ${ }^{3,4}$

Figure 1: Natural Products and Lead molecules possessing a spiroimidazolidinone core.

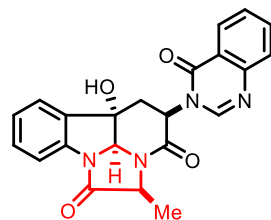

(-)Chaetominine

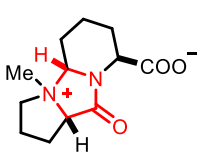

(-)-Dysibetaine PP

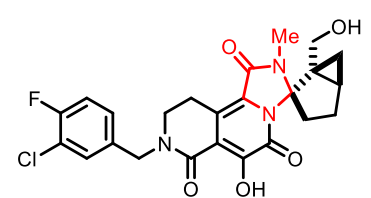

Lead molecule

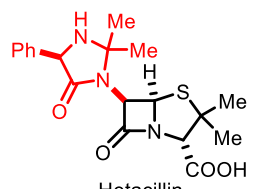

Due to its versatile reactivity, diverse bond formation, and efficiency in chemo- and stereo-selection, organometallic catalysis has always been the preeminent tools to produce chiral molecules. ${ }^{5-9}$ In the last two decades, organocatalysis has emerged as another major tool for enantioselective synthesis. ${ }^{10,11}$ Ever since, domino organocatalysis has been recognized as one of the most efficient strategies to create molecules with multiple stereocenters. ${ }^{12-14}$ Among a plethora of available methods for rapidly building up molecular complexity, oxidative dearomatization is a powerful technique to convert 2-D flat structures into 3-D organic structures in numerous domino organocatalytic processes. ${ }^{15-19}$ Another substantial strategy to create stereochemically rich architectures is operating local desymmetrization under organocatalytic conditions. Engineering an enantiotopic/diasterotopic group discrimination, local desymmetrization rapidly transforms prochiral or meso-molecules into chiral complex molecules with high conformity. ${ }^{20,21}$ Generally, a chiral organometallic or an organocatalyst is an essential part of most reactions designed to create a complex architecture. Therefore, rapid assembly of chiral molecules with multiple stereocenters from simple building blocks under a one-pot metal- and organocatalyst-free condition is a daunting challenge.

Among early development of metal-free synthesis of chiral molecules, MacMillan's pioneer works on iminium/enamine catalysis of chiral imidazolidinones have inspired ample applications of these molecules in numerous asymmetric transformations. ${ }^{22-25}$ With many natural products and pharmaceutical lead molecules possessing a spiroimidazolidinone core, designing a new class of chiral imidazolidinones that possess viable functional groups embedded within their structures for extended elaboration would present a substantial synthetic opportunity.

With its unique symmetrical cyclic structure and rich functionalities, the cyclohexadienone is an attractive template to build complex cyclic architectures. It is beneficial that the substructure can be readily prepared from an oxidative dearomatization reaction of a phenol derivative. In fact, numerous transition metal catalysis and organocatalysis have been utilized for the asymmetric desymmetrization of cyclohexadienones. ${ }^{26-40}$ Recently, aza-Michael/Michael cyclization, a potentially powerful reaction to construct aza-cyclic complex molecules, was designed for the asymmetric desymmetrization of aza-cyclohexadienones. 
In this transformation, diarylprolinol silyl ethers promote the cyclization of para-quinamines with $\alpha, \beta$-unsaturated aldehydes under iminium and enamine catalysis. ${ }^{41}$ The reversible addition of an amine onto iminium species, the substrate/catalyst competitive addition and the intermolecular nature of the strategy are cited as potential challenges to realize this reaction.

In this paper, we aimed to develop a metal-, and organocatalyst-free one-pot assembly of complex spiroimidazolidinone molecules, containing six contiguous stereocenters from simple flat building blocks and a chiral amino acid precursor (scheme 1). To accomplish this task, it is crucial that individual components would be arranged to react and produce chiral advanced intermediates that could engineer the next highly diastereoselective transformations. Allured by a functionality-rich chiral species that could also play a role of a versatile organocatalyst in chemical reactions, we identified a chiral spiroimidazolidinone cyclohexadienone (S) as a special intermediate for our quest to develop a method that can quickly buildup molecular complexity (scheme 1). The aspiration for our research comes from the synthetic accessibility and the special structure/reactivity of a spiroimidazolidinone cyclohexadienone. Structurally, spiroimidazolidinone cyclohexadienone possesses an imidazolidinone template with an azanucleophillic site and a 1,4-cyclohexadienone core. Synthetically, we envisioned that chiral spiroimidazolidinone cyclohexadienones could be prepared directly from amino acids and $N$-alkylated- $p$-aminophenols. Potentially, a chiral spiroimidazolidinone cyclohexadienone could undergo an aza-Michael/Michael cyclization with a Michael acceptor to generate an aza-tricyclic molecule. Overall, we would combine these three components in a one-pot transformation.

Scheme 1. Conceptual design

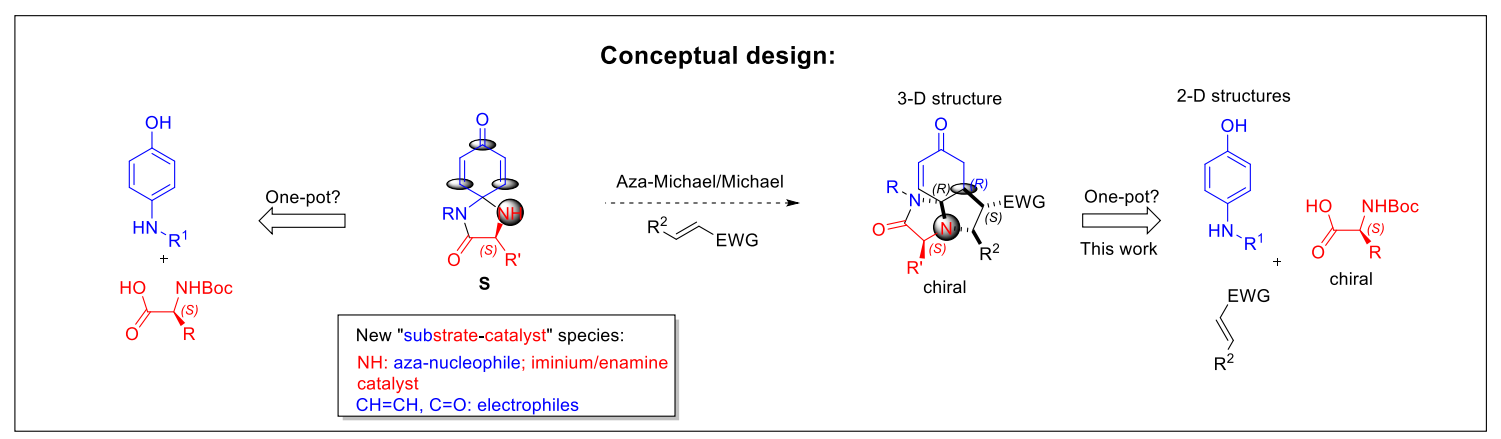

This three-component assembly constructing one C-C and three C-N bonds would produce a distinct 2-D to 3-D shape transforming. Realizing this strategy would present a powerful framework to rapidly create an enantiopure 3-D complex architecture without a metal and a chiral catalyst. However, to realize this chemistry, several substantial challenges need to be addressed. (a) Establish a direct preparation of a chiral spiroimidazolidinone cyclohexadienone from an amino acid and a $N$-alkylated- $p$-aminophenol. This unknown chemistry needs to be developed from the ground up. (b) Realize the aza-Michael/Michael cascade reaction for the spiroimidazolidinone cyclohexadienone and derive a high diastereoselectivity for the overall transformation. We recognized that enabling chemical reactivity of a typically intrinsic weak nucleophile imidazolidinone in an aza-Michael/Michael addition and controlling the formation of six contiguous stereocenters without a metal and a chiral catalyst in a single pot process are both chemically and conceptually challenging. (c) Establish an overall metal-, and organocatalyst-free one pot sequence ensuring that reagents and solvents from previous steps do not interfere with the sequential reactions. It is chemically challenging as many distinctive bond-forming and bond-breaking events would occur during the whole process. 


\section{Results and discussion}

We began our study with a direct preparation of a chiral spiroimidazolidinone cylohexadienone. Deviating from conventional syntheses of chiral imidazolidinones via the condensation of amino amides and carbonyl compounds ${ }^{42}$, we intended to develop a straightforward synthesis of spiroimidazolidinone cyclohexadienones from an amide coupling/oxidative dearomatization sequential reaction of a chiral $N$-Boc-protected amino acid and a $N$-alkylated- $p$-aminophenol. Substrate Ia, prepared from a typical amide coupling reaction of $N$-benzylaminophenol $(\mathbf{B} 1)$ and $N$-Boc-L-phenylalanine (A1), was chosen as our model substrate for the oxidative dearomatization/cyclization sequence. Intriguingly, it is reported that the $N$-protecting group would influence the outcome of an oxidative dearomatization reaction. When an amide or a carbamate (a weak nitrogenic nucleophile) was used in the oxidative dearomatization of a phenol, a competitive ipso addition of oxygen-based (the carbonyl oxygen) nucleophile would prevail that of nitrogen-based nucleophile (the amide or carbamate). ${ }^{43,44}$ Nonetheless, using the Boc-protecting group for the later labile treatment, we hoped that the anilinic nitrogen in Ia would enhance the electrophilicity of the ring and enable a desired nitrogenic-ipso addition of the carbamate-nitrogenic nucleophile.

Scheme 2. Reaction development

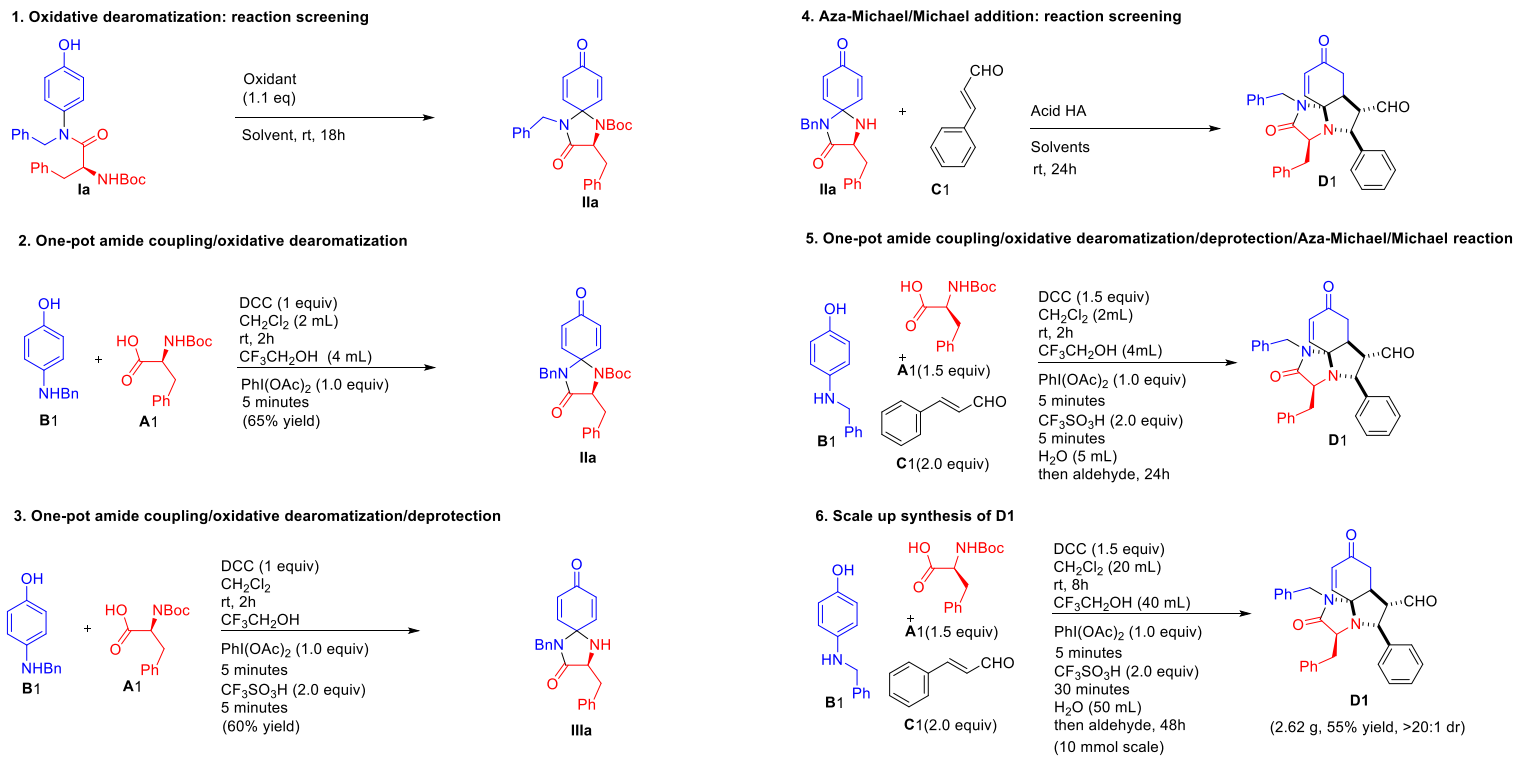

Hence, hypervalent iodines, typical oxidants used in various oxidative dearomatization of phenols, were chosen to investigate for the oxidative dearomatization of Ia (scheme 2, reaction 1). Results were presented in table 1. Whereas most solvents and hypervalent iodine reagents failed to deliver the desired products (table 1, entries 1-5, 7, 10-12), we were able to achieve the oxidative dearomatization using diacetoxyiodo benzene (DIB) in 2,2,2-trifluoroethanol (TFE) (table 1, entry 6,). An addition of urea to the reaction did not affect the outcome of the cascade (table 1, entry 13). While acetonitrile and DMF delivered a low yield reaction (table 1, entry 8,9), the combination of TFE with solvent such as acetonitrile (table 1, entry 14-16) or dichloromethane (DCM) (table 1, entry 17) produced the desired product with satisfied yields. Unfortunately, attempts to achieve a one-pot procedure of amide coupling/oxidative dearomatization in TFE were met with no success. The one-pot procedure was realized when we combined using DCM as the sole solvent in the amide coupling step and a (2:1) TFE/DCM solution in the oxidative dearomatization. Under this condition, imidazolidinone IIa was isolated with $65 \%$ yield (scheme 2, reaction 2). Its structure was unambiguously assigned from the X-ray crystallography. The free amine IIIa was then produced with good yield (60\% yield, scheme 2, reaction 3) under a one-pot amide coupling/oxidative dearomatization/deprotection procedure. 


\begin{tabular}{|c|c|c|c|c|}
\hline entry & oxidant & solvent & additive & yield \\
\hline 1 & $\mathrm{PhI}(\mathrm{OAc})_{2}$ & $\mathrm{Et}_{2} \mathrm{O}$ & None & $0 \%$ \\
\hline 2 & $\mathrm{PhI}(\mathrm{OAc})_{2}$ & $\mathrm{CH}_{2} \mathrm{Cl}_{2}$ & None & $0 \%$ \\
\hline 3 & $\mathrm{PhI}(\mathrm{OAc})_{2}$ & $\mathrm{CHCl}_{3}$ & None & $0 \%$ \\
\hline 4 & $\mathrm{PhI}(\mathrm{OAc})_{2}$ & $\mathrm{THF}$ & None & $0 \%$ \\
\hline 5 & $\mathrm{PhI}(\mathrm{OAc})_{2}$ & $\mathrm{C}_{6} \mathrm{H}_{5} \mathrm{CH}_{3}$ & None & $0 \%$ \\
\hline 6 & $\mathrm{PhI}(\mathrm{OAc})_{2}$ & $\mathrm{CF}_{3} \mathrm{CH}_{2} \mathrm{OH}$ & None & $90 \%$ \\
\hline 7 & $\mathrm{PhI}(\mathrm{OAc})_{2}$ & $\mathrm{CH}_{3} \mathrm{OH}$ & None & $0 \%$ \\
\hline 8 & $\mathrm{PhI}(\mathrm{OAc})_{2}$ & $\mathrm{CH}_{3} \mathrm{CN}$ & None & $35 \%$ \\
\hline 9 & $\mathrm{PhI}(\mathrm{OAc})_{2}$ & $\mathrm{DMF}_{3}$ & None & $25 \%$ \\
\hline 10 & $\mathrm{IBX}$ & $\mathrm{CF}_{3} \mathrm{CH}_{2} \mathrm{OH}$ & None & $0 \%$ \\
\hline 11 & $\mathrm{DMP}$ & $\mathrm{CF}_{3} \mathrm{CH}_{2} \mathrm{OH}$ & None & $0 \%$ \\
\hline 12 & $\mathrm{NaIO} 4$ & $\mathrm{CF}_{3} \mathrm{CH}_{2} \mathrm{OH}$ & None & $0 \%$ \\
\hline 13 & $\mathrm{PhI}(\mathrm{OAc})_{2}$ & $\mathrm{CF}_{3} \mathrm{CH}_{2} \mathrm{OH}$ & Urea & $90 \%$ \\
\hline 14 & $\mathrm{PhI}(\mathrm{OAc})_{2}$ & $\mathrm{TFE}: \mathrm{CH}_{3} \mathrm{CN}(3: 1)$ & Urea & $87 \%$ \\
\hline 15 & $\mathrm{PhI}(\mathrm{OAc})_{2}$ & $\mathrm{TFE}: \mathrm{CH}_{3} \mathrm{CN}(2: 1)$ & Urea & $80 \%$ \\
\hline 16 & $\mathrm{PhI}(\mathrm{OAc})_{2}$ & $\mathrm{TFE}: \mathrm{CH}_{3} \mathrm{CN}(1: 1)$ & Urea & $65 \%$ \\
\hline 17 & $\mathrm{PhI}(\mathrm{OAc})_{2}$ & $\mathrm{TFE}: \mathrm{CH}_{2} \mathrm{Cl}{ }_{2}(2: 1)$ & Urea & $85 \%$ \\
\hline
\end{tabular}

Table 1. Reaction screening for oxidative dearomatization reaction of Ia

This research aims to create six contiguous stereocenters around an aza-tricyclic molecule with a high stereoselectivity. Since the chirality of the amino acid is embedded into the spiroimidazolidinone, the overall stereoselectivity of the process would depend on the stereoselectivity of the aza-Michael/Michael addition of spiroimidazolidinone $\mathbf{S}$ with a Michael acceptor. In the absence of an organometallic or a chiral organocatalyst, controlling the Re/Si facial approach in the aza-Michael addition and both facial and group selectivity of the cyclohexadienone in the following Michael addition would be a tall order. Another challenging aspect of realizing the desired aza-Michael/Michael addition is that an imidazolidinone is typically an intrinsic weak nucleophile. ${ }^{45}$ We envisioned that the lackluster reactivity of an imidazolidinone and the challenging face-selection of the aza-Michael/Michael cascade could be addressed if an $\alpha, \beta$-unsaturated aldehyde was used for this reaction. Unlike other Michael acceptors, an $\alpha, \beta$ unsaturated aldehyde would be activated by forming an iminium with the imidazolidinone intermediate $\mathbf{S}$. The amplified electrophilicity of the iminium would accommodate for the low nucleophilicity of the imidazolidinone, ${ }^{46}$ allowing its participation in a sequential aza-Michael/Michael cascade reaction as both a substrate and a chiral iminium/enamine catalyst. This substratecatalyst (sub-catalyst) dual role of the imidazolidinone would enhance a co-ordinational proximity of the chiral substrate and the catalyst in the transition state and magnify the steric discrimination of facial attacks.

\footnotetext{
${ }^{*}$ Indeed, in a quick survey of the cyclization of amine IIIa with Michael acceptors such as nitro styrene, (E)-chalcone and Ethyl $\beta$ - $(p-\mathrm{MeO}$-phenyl)acrylate, no reaction was observed under these conditions (for experiment details, see table 2 of the supporting information).
} 
Scheme 3. Mechanistic proposal for the Aza-Michael/Michael addition/desymmetrization

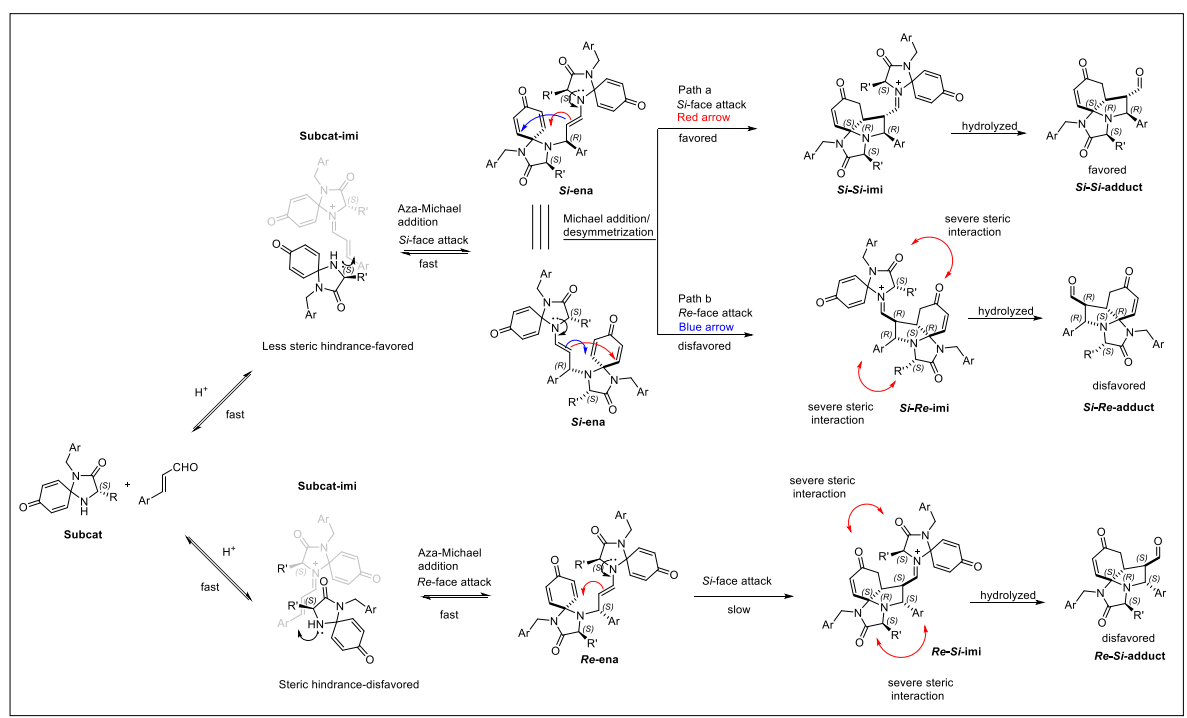

A mechanistic scenario for the reaction of a (S)-imidazolidinone is depicted in scheme 3. The imidazolidinone Subcat would approach the Subcat-imi from both $R e$-face and Si-face with a fast reversible aza-Michael/retro aza-Michael process. With R' group from the catalyst obstructing Subcat from the $R e$-face of the iminium, the favored $\mathrm{Si}$-face attack would produce the enamine Si-ena. This enamine would then undergo an intramolecular Michael addition to one of the two diastereotopic vinylic ketones either from Si-face (path a, red arrow) forming Si-Si-imi, or Re-face (path b, blue arrow), forming Si-Re-imi, under an enamine catalysis. This $R e$-face attack to form Si-Re-imi would be highly disfavored as it suffers from two significant steric interactions: the developing steric interaction of $\mathrm{R}^{\prime}$ from in the substrate with the ring-forming pyrrolidine, and the interaction of the bulky catalyst site with the cyclohexenone core of the adduct. On the other hand, the Si-face attack to form Si-Si-imi would avoid these steric cumbers and eventually produce $\mathbf{S i - S i - a d d u c t . ~ T h i s ~ d i a s t e r e o t o p i c ~ s e l e c t i o n ~ ( p a t h ~ a ~ o v e r ~ p a t h ~ b ) ~ l o c k s ~ t h e ~ n i t r o g e n ~ i n ~ a ~ r i g i d ~}$ aza-tricyclic framework and concurrently operates a desymmetrization of the cyclohexadienone core. Nevertheless, the unfavored $R e$-face attack of Subcat onto the iminium Subcat-imi would produce enamine Re-ena. Supposedly, the intramolecular Michael addition to one of the two diastereotopic vinylic ketones of the cyclohexadienone would favor the Si-face attack. However, the formation of Re-Si-imi would still suffer from the steric interactions of the arene ring of the aldehyde with the imidazolidione ring of the substrate as well as the bulky catalyst site with the cyclohexanone ring of the substrate. With a fast reversible azaMichael/retro aza-Michael, Re-Si-ena would effectively be a reservoir of non-reactive aggregates due to its unfavored formation and unfavored forward Michael reaction to Re-Si-imi. The Curtin-Hammett kinetics would eventually drive the balance toward the formation of Si-Si-adduct. Overall, this feature would induce a desired reactivity and stereoselectivity for the construction of only the enantiomer Si-Si-adduct. Essentially, this aza-Michael/Michael addition cascade would define the simultaneous formation of the five new stereogenic centers including stereochemically challenging features such as a spiroaminal and a nitrogenic stereocenter. Supposedly, a $(R)$-imidazolidinone would produce the enantiomer of Si-Si-adduct.

The task then was to realize a suitable condition for the aza-Michael/Michael cascade of the spiroimidazolidinone and an $\alpha, \beta$ unsaturated aldehyde and establish a combined overall one-pot condition for a series of distinctive reactions amide coupling/oxidative dearomatization/cyclization/deprotection/aza-Michael/Michael addition. When a mixture of amine IIIa and (E)cinnamaldehyde $(\mathbf{C} 1)$ in DCM for 24 hours showed no reaction, several Bronsted acids were probed to promote the azaMichael/Michael cascade (table 2). ${ }^{47}$ 
Table 2. Reaction screening for Aza-Michael/Michael reaction

\begin{tabular}{|c|c|c|c|c|}
\hline entry & acid HA & solvent & yield & $\mathrm{dr}$ \\
\hline 1 & none & DCM & $0 \%$ & nd \\
\hline 2 & TFA & DCM & $90 \%$ & $>20: 1$ \\
\hline 3 & PTSA & DCM & $0 \%$ & nd \\
\hline 4 & Triflic acid & DCM & $0 \%$ & nd \\
\hline 5 & AcOH & DCM & $35 \%$ & $>20: 1$ \\
\hline 6 & $\mathrm{HCl} 37 \%$ & DCM & $0 \%$ & nd \\
\hline 7 & $\mathrm{HBr} 49 \%$ & DCM & $0 \%$ & nd \\
\hline 8 & $\mathrm{H}_{2} \mathrm{SO}_{4}$ & DCM & $0 \%$ & nd \\
\hline 9 & $\mathrm{H}_{3} \mathrm{PO}_{4}$ & DCM & $0 \%$ & nd \\
\hline 10 & $\mathrm{TFA}^{2}$ & DCM/TFE $(1: 2)$ & $0 \%$ & nd \\
\hline
\end{tabular}

Complex mixture with no desired product were observed in most cases (table 2, entries 1,3-4, 6-10). Only TFA (table 2 entry 2) and acetic acid ( $\mathrm{AcOH})$ (table 2 entry 5) delivered the desired product with excellent diastereoselectivity ( $>20: 1 \mathrm{dr})$. TFA proved to be superior as it produced an excellent outcome (90\% yield, >20:1 dr). Surprisingly, the reaction of IIIa and (E)-cinnamaldehyde (C1, 2 equiv) with TFA (2 equiv) in mixture of DCM/TFE (1:2) solution did not produce D1 (table 2, entry 10).

Whereas triflic acid (2 equivalents) in DCM/TFE (1:2) solution allowed the Boc-deprotection of IIa to IIIa, a one-pot reaction of IIa with triflic acid (2 equivalents) in DCM/TFE (1:2) solution with (E)-cinnamaldehyde (C1) only produced complex mixtures with no desired product D1. Quenching acid excess with weak bases such as triethyl amine or solid sodium bicarbonate before adding $\mathbf{C} 1$ still only generated a complex mixture. Apparently, the desired one-pot condition must alleviate the undesired role of the acid excess and TFE. We noticed that, unlike most of metal-catalyzed reactions, water can sometimes be added to an organocatalyzed reaction. ${ }^{48}$ We envisioned that, adding water to the reaction would create a two-phase solution where aqueous phase would extract the excess of acids and TFE, while the organic phase (DCM) would contain protonated amine with the aldehyde. The protonated aza-tricyclic adduct could also play a role of a phase-transfer catalyst in this two-phase condition and accelerate the aza-Michael/Michael cyclization. Satisfyingly, treatment of IIa with triflic acid (2 equiv) in DCM for five minutes before the addition of $\mathrm{H}_{2} \mathrm{O}$ and a solution of $\mathbf{C} 1$ in DCM eventually produced $\mathbf{D} 1$ with $75 \%$ yield and >20:1 dr. Surprisingly, when IIIa was mixed with $\mathrm{C} 1$ and TFA, the treatment of water immediately shut down the reaction.

We are pleased to establish a one-pot procedure using of $N$-Boc-L-phenylalanine (A1), aminophenol (B1), (E)-cinnamaldehyde (C1) and triflic acid in an aqueous-based condition (scheme 2, reaction 5). Under this optimal condition, D1 was produced with moderate yield (55\%) and excellent diastereoselectivity (>20:1).

\footnotetext{
${ }^{\dagger}$ Only $25 \%$ conversion to the desired cyclization adduct was observed when the mixture was stirred at room temperature for 7 days.
} 
With the optimized condition in hand, the scope of $N$-substituted aminophenols was first explored (D1-10, scheme 4). Substrates with either electron-donating or electron-withdrawing groups produced desired products with satisfied yields. The substituents in the phenyl ring of the substrates do not exhibit a significant effect on the reaction as we observed only a slightly drop in the reaction yield of ortho-methyl substitution (D2, scheme 4). Additionally, the reaction was tolerated with halogens at various positions in the phenyl ring of the amine (D6-D10, scheme 4). Next, the scope of $\alpha, \beta$-unsaturated aldehydes was examined. The reaction delivered the desired products with consistent moderate yields and >20:1 $\mathrm{dr}$ (D11-16, scheme 4). Other protected $\alpha$-amino acids were then investigated (D17-23, scheme 4). $N$-Boc- $\alpha$-amino acids such as $N$-Boc-L-valine (D18), O-Benzyl- $N$-Boc-L-tyrosine (D19), $N$-Boc-L-isoleucine (D21), O-benzyl- $N$-Boc-L-threonine (D22) consistently produced the desired products with moderate to good yields and >20:1 dr. For substrates such as $N$-Boc-L-alanine (D17) and N-Boc-L-leucine (D20), we found that a two-step procedure was necessary to achieve the desired products with excellent diastereoselectivity as the one-pot only produced a complex mixture. In fact, after the Boc-deproctection step, the reaction was quenched with aqueous $\mathrm{NaHCO}_{3}$ solution and extracted in DCM followed by solvents removal. The crude mixture was then carried on with the aza-Michael/Michael addition in DCM with TFA (see supporting information for details). Interestingly, when we employed $N$-Boc-D-phenylalanine in the one-pot condition with $\mathbf{C} 1$ and $\mathbf{B} 1$, the reaction produced $\mathbf{D} 23$, an enantiomer of $\mathbf{D} 1$, with an equal efficiency (55\% yield, >20:1 dr). (Z)-cinnamaldehyde was used in either the one-pot procedure or the TFA-catalyzed aza-Michael/Michael addition with the amine IIIa. However, in contrast to our expectation that a diastereomer of $\mathbf{D} 1$ would be generated, no other diastereomers was observed in the reaction and D1 was always the only cyclization adduct (see supporting information for details). We noted that, under the reaction condition, the (Z)-cinnamaldehyde was completely converted to (E)-isomer before any cyclization event. This result demonstrates a fast, reversible aza-Michael/retro aza-Michael process. Unfortunately, preliminary screening showed that the one-pot reaction only works well with cinnamaldehyde derivatives as a one-pot reaction of $\mathbf{A} 1, \mathbf{B} 1$ with (E)-crotonaldehyde gave a complex mixture. The relative structural configuration of products from our proposed mechanism was supported by NOESY NMR of D15 (see supporting information for details). 
Scheme 4. Scope of the reaction

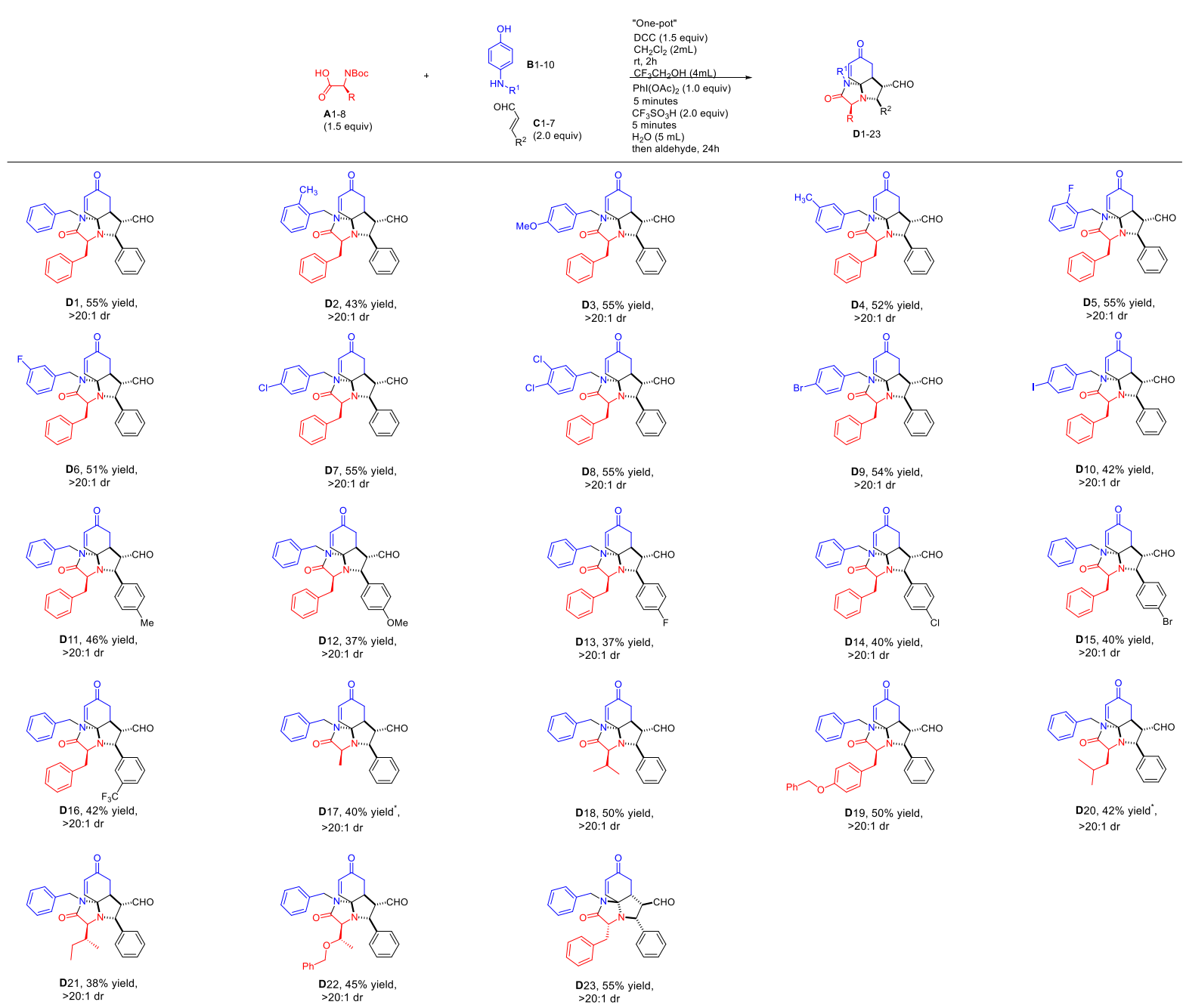

To further highlight the potential applicability of this methodology, a one-pot reaction was carried out at $10 \mathrm{mmol}$ scale. When A1,

$\mathbf{B} 1$ and $\mathbf{C} 1$ were subjected to the one-pot procedure with technical grade reagents under ambient atmosphere, $2.62 \mathrm{~g}$ of the desired product D1 was isolated with 55\% yield and excellent dr (>20:1 dr) (scheme 2, reaction 6).

\section{Mechanistic investigation}

To probe into the proposed iminium/enamine catalysis, a spectroscopic profile of reaction of three mixtures at ratio of IIIa/Ecinnamaldehyde corresponding to (1.0:15.0); (1.0:1.0); (1.5:1.0) and TFA in $\mathrm{CDCl}_{3}$ was monitored (for full details of these experiments, see table 4 in the supporting information). In the mass spectroscopy of the crude reaction measured at 30 minutes, the molecular peak of the iminium at $\mathrm{m} / \mathrm{z}=459.2068$ is observed at peak with $\mathrm{m} / \mathrm{z}=459.1975$. ${ }^{1} \mathrm{H}-\mathrm{NMR}$ spectrum of each mixture was compared with ${ }^{1} \mathrm{H}-\mathrm{NMR}$ spectra of IIIa, E-cinnamaldehyde and D1. The absence of an aldehyde peak from a direct azaMichael addition of IIIa onto E-cinnamaldehyde effectively rules out a direct Brønsted acid-catalyzed mechanism. While it is not possible to isolate the iminium and enamine intermediates, the iminium/enamine formation was observed with some distinctive peaks in ${ }^{1} \mathrm{H}-\mathrm{NMR}$. Treatment of the reaction mixture with water after one-hour, we obtained a mixture of free amine IIIa, the unreactive (E)-cinnamaldehyde (C1) and a small portion ( $~ 8 \%$ conversion) of cyclization adduct D1 (Figure 2, for more details see the supporting information). The recovery of IIIa and $\mathbf{C 1}$ from the reaction mixture implies an acid-catalyzed iminium/Michael 
addition/enamine formation-hydrolysis/retro aza-Michael addition transformation. To uncover more concrete proof for the iminium formation, we envisioned that a competitive interruptive reduction of the transient iminium with a weak reducing agent would produce an isolable intermediate for characterization. Indeed, a reaction mixture of the amine IIIa (1.0 equiv), (E)-cinnamaldehyde (2.0 equiv) and TFA ( 2 equiv) in chloroform was treated with $\mathrm{NaBH}(\mathrm{OAc})_{3}$ at room temperature for 18h (scheme 5). While we, surprisingly, did not observe the formation of the reduced tertiary amine $\mathbf{X}$, we were able to isolate imine $\mathbf{Y}$ with $25 \%$ yield (in 1:2 mixture with the aza-Michael/Michael addition adduct D1). The exclusive formation of $\mathbf{Y}$ over $\mathbf{X}$ showcased that imidazoleiminium is exclusively reduced at the aminal center. No reaction was observed when a mixture of amine IIIa, TFA and $\mathrm{NaBH}(\mathrm{OAc})_{3}$ in chloroform was stirred at room temperature for $18 \mathrm{~h}$. Under this condition, we fully recovered the amine IIIa. This controlled experiment rules out a pathway of formation of $\mathbf{Y}$ from the condensation of $\mathbf{C} 1$ and amine $\mathbf{W}$. The isolation of amine $\mathbf{Y}$ confirmed our proposed hypothesis that the iminium Subcat-imi was formed during the course of reaction.

Figure 2. NMR experiments

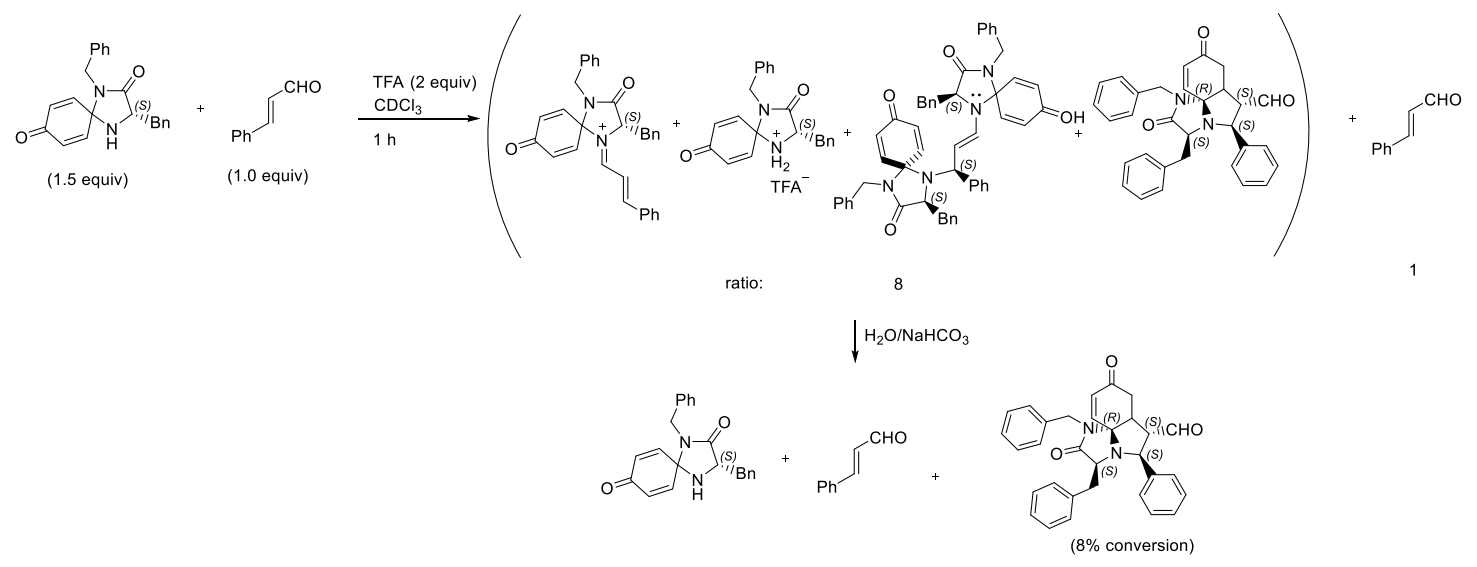

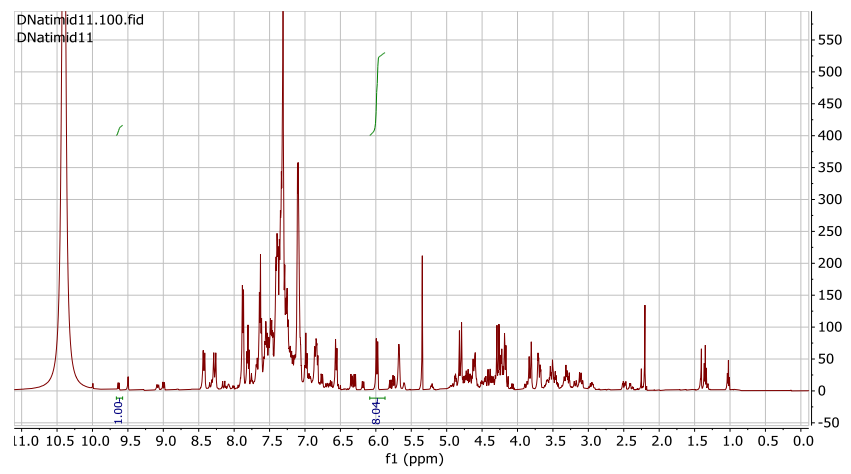

Reaction mixture: IIIa (1.5 equiv) + (E)-cinnamldehyde)

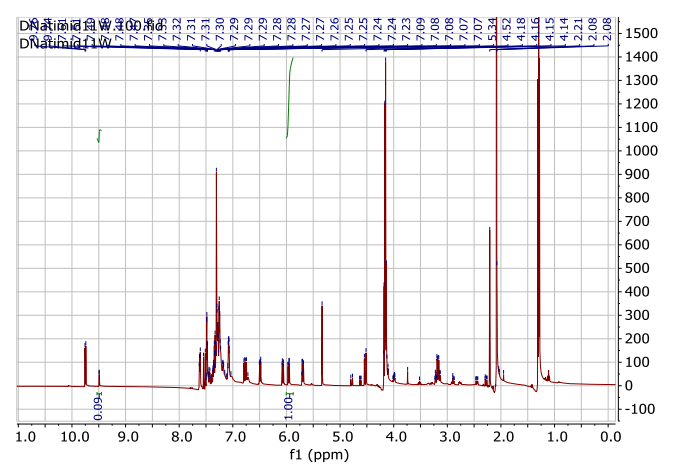

After treatment with water: IIIa $+($ E)-cinnamaldehyde + D1 
Scheme 5. Intercept reduction

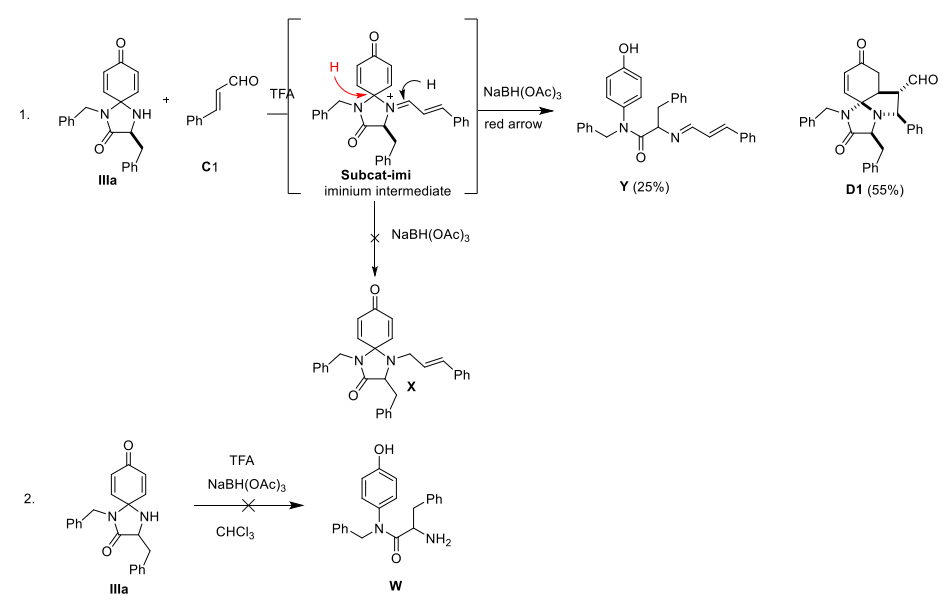

\section{A hidden sub-catalysis}

Without a transition metal or an organocatalyst, the aza-tricyclic molecules were directly assembled from $N$-alkylated aminophenols, $\alpha, \beta$-unsaturated aldehydes and chiral $\alpha$-amino acids with excellent stereo control. Apparently, no conventional catalysis would be accurately described for the overall transformation. Whereas the spiroimidazolidinone cylohexadienone intermediate does promote the aza-Michael/Michael cascade, it does not entirely fit into a classic definition of a catalyst. Characteristically, a catalyst is introduced to catalyze a reaction without itself undergoing any permanent chemical change. Spiroimidazolidinone $\mathbf{S}$, however, is only temporary created and eventually consumed to form the final product. Conceptually, we characterized the spiroimidazolidinone as a sub-catalyst. The sub-catalyst conceptual also represents its dual role of a substrate and a catalyst in the aza-Michael/Michael cascade reaction. Driven by these characteristics of the spiroimidazolidione sub-catalyst, the hidden sub-catalysis allows the use of an amino acid precursor as the sole chirality genesis to control the formation of six contiguous stereocenters of aza-tricylic molecules in a one-pot procedure.

\section{Conclusion and outlook}

We have demonstrated a practical and scalable rapid assembly of chiral aza-tricyclic molecules containing six contiguous stereocenters with moderate to good yield and a universal excellent diastereoselectivity ( $>20: 1 \mathrm{dr}$ ). Apart from the tertiary stereogenic center originated from the amino acid precursor, all the five newborn stereogenic centers, including stereochemically challenging features such as a spiroaminal and a nitrogenic stereogenic centers, were constructed with excellent stereocontrol in tandem of the aza-Michael/Michael addition. Whereas no transition metals- or an organocatalyst was used, the strategically produced imidazolidinone intermediate displays a hidden substrate-catalyst dual role to promote sequential self-catalyzed reactions. Overall, the stereoselectivity of the whole process is set up only by the chirality of the amino acid precursor. Whereas the products were prepared with moderate to good yield, the efficiency of the process is inadvertently disguised as this one-pot procedure involves five bond-forming events in a series of distinctive reactions amide coupling/oxidative dearomatization/cyclization/deprotection/aza-Michael/Michael addition. The reaction is highly efficient both in the number of newly formed bonds and the increased degree of bulkiness in a single pot transformation. The use of commercial enantiopure amino acids allows the preparation of enantiopure aza-tricyclic molecules. The abundant availability of both natural and unnatural amino 
acids chiral pool will offer unrestricted chirality sources for this transformation. With readily available precursors and their multitude combinations, this three-component assembly can offer a large library of aza-tricyclics. These aza-tricyclic adducts possess many distinctive functional groups for structural elaboration for potential usages.

Acknowledgements: The author is grateful to Mac Group at Vietnam National University (VNU) and Wright lab at the University of Connecticut (UCONN) for sharing lab facility. The author thanks Dr Son Nguyen (VNU) for his assistance on the HRMS measurement of compounds and Dr. Thang Pham (VNU) for the X-ray crystallography of compound IIa. The author thanks Professor Jeffrey S. Johnson (University of North Carolina at Chapel Hill) for bringing up Curtin-Hammett kinetics mechanism discussion during the manuscript preparation. The author is grateful to Dr. Linh Nguyen (Bates College) for proofreading the manuscript and supporting the pursue of this research. 


\section{References:}

(1) Mohr, J. T.; Krout, M. R.; Stoltz, B. M. Natural Products as Inspiration for the Development of Asymmetric Catalysis. Nature 2008, 455 (7211), 323-332. https://doi.org/10.1038/nature07370.

(2) Furst, L.; Stephenson, C. R. J. Nature-Inspired Total Synthesis. Nat. Chem. Biol. 2011, 7 (9), 582-583. https://doi.org/10.1038/nchembio.637.

(3) Hönig, M.; Sondermann, P.; Turner, N. J.; Carreira, E. M. Enantioselective Chemo- and Biocatalysis: Partners in Retrosynthesis. Angew. Chem. Int. Ed. 2017, 56 (31), 8942-8973. https://doi.org/10.1002/anie.201612462.

(4) Taylor, M. S.; Jacobsen, E. N. Asymmetric Catalysis in Complex Target Synthesis. Proc. Natl. Acad. Sci. U. S. A. 2004, 101 (15), 5368. https://doi.org/10.1073/pnas.0307893101.

(5) Toste, F. D.; You, S.-L. Asymmetric Synthesis Enabled by Organometallic Complexes. Organometallics 2019, 38 (20), 3899-3901. https://doi.org/10.1021/acs.organomet.9b00627.

(6) Zhou, Q.-L. Transition-Metal Catalysis and Organocatalysis: Where Can Progress Be Expected? Angew. Chem. Int. Ed. 2016, 55 (18), 5352-5353.

https://doi.org/10.1002/anie.201509164.

(7) Vargová, D.; Némethová, I.; Plevová, K.; Šebesta, R. Asymmetric Transition-Metal Catalysis in the Formation and Functionalization of Metal Enolates. ACS Catal. 2019, 9 (4), 3104-3143. https://doi.org/10.1021/acscatal.8b04357.

(8) Cherney, A. H.; Kadunce, N. T.; Reisman, S. E. Enantioselective and Enantiospecific Transition-Metal-Catalyzed Cross-Coupling Reactions of Organometallic Reagents To Construct C-C Bonds. Chem. Rev. 2015, 115 (17), 9587-9652.

https://doi.org/10.1021/acs.chemrev.5b00162.

(9) Blaser, H.-U. Industrial Asymmetric Catalysis: Approaches and Results. Rendiconti Lincei 2007, 18 (4), 281-304. https://doi.org/10.1007/BF02934925.

(10) MacMillan, D. W. C. The Advent and Development of Organocatalysis. Nature 2008, 455 (7211), 304-308. https://doi.org/10.1038/nature07367.

(11) Xiang, S.-H.; Tan, B. Advances in Asymmetric Organocatalysis over the Last 10 Years. Nat. Commun. 2020, 11 (1), 3786. https://doi.org/10.1038/s41467-020-17580-z.

(12) Grondal, C.; Jeanty, M.; Enders, D. Organocatalytic Cascade Reactions as a New Tool in Total Synthesis. Nat. Chem. 2010, 2 (3), 167-178. https://doi.org/10.1038/nchem.539.

(13) Enders, D.; Hüttl, M. R. M.; Grondal, C.; Raabe, G. Control of Four Stereocentres in a Triple Cascade Organocatalytic Reaction. Nature 2006, 441 (7095), 861-863. https://doi.org/10.1038/nature04820.

(14) Vetica, F.; de Figueiredo, R. M.; Orsini, M.; Tofani, D.; Gasperi, T. Recent Advances in Organocatalytic Cascade Reactions toward the Formation of Quaternary Stereocenters. Synthesis 2015, 47 (15), 2139-2184.

(15) Roche, S. P.; Porco, J. A., Jr. Dearomatization Strategies in the Synthesis of Complex Natural Products. Angew. Chem. Int. Ed Engl. 2011, 50 (18), 4068-4093. https://doi.org/10.1002/anie.201006017.

(16) Reddy, C. R.; Prajapti, S. K.; Warudikar, K.; Ranjan, R.; Rao, B. B. Ipso-Cyclization: An Emerging Tool for Multifunctional Spirocyclohexadienones. Org. Biomol. Chem. 2017, 15 (15), 3130-3151. https://doi.org/10.1039/C7OB00405B. 
(17) Pouységu, L.; Deffieux, D.; Quideau, S. Hypervalent Iodine-Mediated Phenol Dearomatization in Natural Product Synthesis. Tetrahedron 2010, 66 (13), 2235-2261. https://doi.org/10.1016/j.tet.2009.12.046.

(18) Vo, N. T.; Pace, R. D. M.; O’Har, F.; Gaunt, M. J. An Enantioselective Organocatalytic Oxidative Dearomatization Strategy. J. Am. Chem. Soc. 2008, 130 (2), 404-405. https://doi.org/10.1021/ja077457u.

(19) Baker Dockrey, S. A.; Lukowski, A. L.; Becker, M. R.; Narayan, A. R. H. Biocatalytic Site- and Enantioselective Oxidative Dearomatization of Phenols. Nat. Chem. 2018, 10 (2), 119-125. https://doi.org/10.1038/nchem.2879.

(20) Horwitz, M. A.; Johnson, J. S. Local Desymmetrization through Diastereotopic Group Selection: An Enabling Strategy for Natural Product Synthesis. Eur. J. Org. Chem. 2017, 2017 (11), 1381-1390. https://doi.org/10.1002/ejoc.201601481.

(21) Borissov, A.; Davies, T. Q.; Ellis, S. R.; Fleming, T. A.; Richardson, M. S. W.; Dixon, D. J. Organocatalytic Enantioselective Desymmetrisation. Chem. Soc. Rev. 2016, 45 (20), 5474-5540. https://doi.org/10.1039/C5CS00015G.

(22) Paras, N. A.; MacMillan, D. W. C. New Strategies in Organic Catalysis: The First Enantioselective Organocatalytic Friedel-Crafts Alkylation. J. Am. Chem. Soc. 2001, 123 (18), 4370-4371. https://doi.org/10.1021/ja015717g.

(23) Erkkilä, A.; Majander, I.; Pihko, P. M. Iminium Catalysis. Chem. Rev. 2007, 107 (12), 5416-5470. https://doi.org/10.1021/cr068388p.

(24) Lelais, G.; MacMillan, D. Modern Strategies in Organic Catalysis: The Advent and Development of Iminium Activation. Aldrichimica Acta 2006, 39, 79-87.

(25) Deepa; Singh, S. Recent Development of Recoverable MacMillan Catalyst in Asymmetric Organic Transformations. Adv. Synth. Catal. 2020, $n / a$ (n/a). https://doi.org/10.1002/adsc.202000994.

(26) Imbos, R.; Minnaard, A. J.; Feringa, B. L. A Highly Enantioselective Intramolecular Heck Reaction with a Monodentate Ligand. J. Am. Chem. Soc. 2002, 124 (2), 184-185. https://doi.org/10.1021/ja017200a.

(27) Hayashi, Y.; Gotoh, H.; Tamura, T.; Yamaguchi, H.; Masui, R.; Shoji, M. CysteineDerived Organocatalyst in a Highly Enantioselective Intramolecular Michael Reaction. $J$. Am. Chem. Soc. 2005, 127 (46), 16028-16029. https://doi.org/10.1021/ja055740s.

(28) Liu, Q.; Rovis, T. Asymmetric Synthesis of Hydrobenzofuranones via Desymmetrization of Cyclohexadienones Using the Intramolecular Stetter Reaction. J. Am. Chem. Soc. 2006, 128 (8), 2552-2553. https://doi.org/10.1021/ja058337u.

(29) Rubush, D. M.; Morges, M. A.; Rose, B. J.; Thamm, D. H.; Rovis, T. An Asymmetric Synthesis of 1,2,4-Trioxane Anticancer Agents via Desymmetrization of Peroxyquinols through a Brønsted Acid Catalysis Cascade. J. Am. Chem. Soc. 2012, 134 (33), 1355413557. https://doi.org/10.1021/ja3052427.

(30) Gu, Q.; You, S.-L. Desymmetrization of Cyclohexadienones Viacinchonine Derived Thiourea-Catalyzed Enantioselective Aza-Michael Reaction and Total Synthesis of (-)Mesembrine. Chem. Sci. 2011, 2 (8), 1519-1522. https://doi.org/10.1039/C1SC00083G.

(31) Breuning, M.; Corey, E. J. Catalytic Enantioselective Diels-Alder Reactions of 1,4Quinone Monoketals. Org. Lett. 2001, 3 (10), 1559-1562. https://doi.org/10.1021/ol015852y. 
(32) Corbett, M. T.; Johnson, J. S. Enantioselective Synthesis of Hindered Cyclic Dialkyl Ethers via Catalytic Oxa-Michael/Michael Desymmetrization. Chem. Sci. 2013, 4 (7), 2828-2832. https://doi.org/10.1039/C3SC51022K.

(33) Leon, R.; Jawalekar, A.; Redert, T.; Gaunt, M. J. Catalytic Enantioselective Assembly of Complex Molecules Containing Embedded Quaternary Stereogenic Centres from Simple Anisidine Derivatives. Chem. Sci. 2011, 2 (8), 1487-1490.

https://doi.org/10.1039/C1SC00218J.

(34) Keilitz, J.; Newman, S. G.; Lautens, M. Enantioselective Rh-Catalyzed Domino Transformations of Alkynylcyclohexadienones with Organoboron Reagents. Org. Lett. 2013, 15 (5), 1148-1151. https://doi.org/10.1021/ol400363f.

(35) Jia, M.-Q.; You, S.-L. Desymmetrization of Cyclohexadienones Viad-Camphor-Derived Triazolium Salt Catalyzed Intramolecular Stetter Reaction. Chem. Commun. 2012, 48 (51), 6363-6365. https://doi.org/10.1039/C2CC32783J.

(36) Jia, M.-Q.; Liu, C.; You, S.-L. Diastereoselective and Enantioselective Desymmetrization of $\alpha$-Substituted Cyclohexadienones via Intramolecular Stetter Reaction. J. Org. Chem. 2012, 77 (23), 10996-11001. https://doi.org/10.1021/jo3022555.

(37) Wu, W.; Li, X.; Huang, H.; Yuan, X.; Lu, J.; Zhu, K.; Ye, J. Asymmetric Intramolecular Oxa-Michael Reactions of Cyclohexadienones Catalyzed by a Primary Amine Salt. Angew. Chem. Int. Ed. 2013, 52 (6), 1743-1747. https://doi.org/10.1002/anie.201206977.

(38) Kalstabakken, K. A.; Harned, A. M. Asymmetric Transformations of Achiral 2,5Cyclohexadienones. Tetrahedron 2014, 70 (51), 9571-9585. https://doi.org/10.1016/j.tet.2014.07.081.

(39) Shu, T.; Cossy, J. Asymmetric Desymmetrization of Alkene-, Alkyne- and AlleneTethered Cyclohexadienones Using Transition Metal Catalysis. Chem. Soc. Rev. 2021. https://doi.org/10.1039/D0CS00666A.

(40) Al-Tel, T. H.; Srinivasulu, V.; Ramanathan, M.; Soares, N. C.; Sebastian, A.; Bolognesi, M. L.; Abu-Yousef, I. A.; Majdalawieh, A. Stereocontrolled Transformations of Cyclohexadienone Derivatives to Access Stereochemically Rich and Natural ProductInspired Architectures. Org. Biomol. Chem. 2020, 18 (42), 8526-8571. https://doi.org/10.1039/D0OB01550D.

(41) Pantaine, L.; Coeffard, V.; Moreau, X.; Greck, C. Enantioselective Desymmetrization of Para-Quinamines through an Aminocatalyzed Aza-Michael/Cyclization Cascade Reaction. Org. Lett. 2015, 17 (15), 3674-3677. https://doi.org/10.1021/acs.orglett.5b01595.

(42) Blackmore, T.; Thompson, P. IMIDAZOLIDIN-4-Ones: Their Syntheses and Applications. Heterocycles 2011, 83 (9), 1953-1975. https://doi.org/10.3987/REV-11-707.

(43) Kita, Y.; Tohma, H.; Kikuchi, K.; Inagaki, M.; Yakura, T. Hypervalent Iodine Oxidation of N-Acyltyramines: Synthesis of Quinol Ethers, Spirohexadienones, and Hexahydroindol-6-Ones. J. Org. Chem. 1991, 56 (1), 435-438. https://doi.org/10.1021/jo00001a082.

(44) Ciufolini, M. A.; Braun, N. A.; Canesi, S.; Ousmer, M.; Chang, J.; Chai, D. Oxidative Amidation of Phenols through the Use of Hypervalent Iodine Reagents: Development and Applications. Synthesis 2007, 2007 (24), 3759-3772. https://doi.org/10.1055/s-2007990906.

(45) An, F.; Maji, B.; Min, E.; Ofial, A. R.; Mayr, H. Basicities and Nucleophilicities of Pyrrolidines and Imidazolidinones Used as Organocatalysts. J. Am. Chem. Soc. 2020, 142 (3), 1526-1547. https://doi.org/10.1021/jacs.9b11877. 
(46) Mayr, H.; Lakhdar, S.; Maji, B.; Ofial, A. R. A Quantitative Approach to Nucleophilic Organocatalysis. Beilstein J. Org. Chem. 2012, 8, 1458-1478. https://doi.org/10.3762/bjoc.8.166.

(47) Xu, Z.; Buechler, T.; Wheeler, K.; Wang, H. A Three-Component Reaction Based on a Remote-Group-Directed Dynamic Kinetic Aza-Michael Addition: Stereoselective Synthesis of Imidazolidin-4-Ones. Chem. - Eur. J. 2010, 16 (10), 2972-2976. https://doi.org/10.1002/chem.200903508.

(48) van der Helm, M. P.; Klemm, B.; Eelkema, R. Organocatalysis in Aqueous Media. Nat. Rev. Chem. 2019, 3 (8), 491-508. https://doi.org/10.1038/s41570-019-0116-0. 